\title{
Bounded rationality revisited: Making sense of complexity in applied sport science
}

\author{
Sam Robertson ${ }^{1}$, David Joyce ${ }^{2}$ \\ ${ }^{1}$ Institute for Health and Sport (iHeS), Victoria University, Australia \\ ${ }^{2}$ Greater Western Sydney Giants
}

\begin{abstract}
Sport science's accelerated uptake of technology and the resultant data growth has enabled new light to be shed on many of its most complex problems. However, this in turn has led to varying interpretation of these problems and often disagreement across sport science disciplines. This article proposes that revisiting the theory of bounded rationality as it pertains to applied sport science can provide a framework for understanding, accepting and utilising this complexity in judgement and decision-making contexts. Bounded rationality contends that individuals who intend to make rational decisions are bound to make merely satisfactory choices, rather than maximizing or optimising ones. Here, the theory is discussed with respect to how it enables for differences in human judgements to be elucidated based on interindividual variations in information sampling and processing. As data generated from technology in sport continues to grow, these differences are likely to continually increase. The systematic nature of the differences between humans and machines are also explained. Adoption of a bounded rationality approach in applied sports environments can help to understand why differences exist in the interpretation of some of sport science's most complex problems, as well as provide a framework for progressing a collective understanding of these areas.
\end{abstract}

\section{Keywords (five)}

Decision-making, psychology, sports management, technology, computer science

\section{DOI}

http://dx.doi.org/10.31236/osf.io/yh38j (add exact DOI after acceptance)

\section{Citation}

Robertson, S.J., \& Joyce, D. (2019). Bounded rationality revisited: making sense of complexity in applied sport science. SportRxiv. doi: 10.31236/osf.io/yh38j

\section{INTRODUCTION}

Sporting environments are complex. As the volume of accessible data relating to these environments grows, improved insights are afforded into many of sport's most important, pervasive and complex topics such as 
Bounded rationality revisited: Making sense of complexity in applied sport science spaces

training, athlete evaluation and injury risk. This increased information combined with the heightened attention placed on these issues has the potential to lead to multiple avenues of interpretation and therefore considerable disagreement across sport science disciplines. Whilst such disagreement is possibly unavoidable and arguably even desirable, understanding the underlying theoretical underpinnings as to why it exists is required in order for meaningful improvements to be made in both research and practice.

Developed by Herbert Simon [1] and popularised by Daniel Kahneman [2] and Gerd Gigerenzer [3], bounded rationality posits that in complex situations, individuals who intend to make rational decisions are bound to make merely satisfactory choices, rather than maximizing or optimising ones [3,4]. This is because rationality is constantly bounded by interactions of both cognitive and environment constraints [5]. Cognitive constraints include knowledge limitations, level of information processing capacity, conscious or unconscious biases, or even ego. Environmental constraints include the time or resources available to the decision maker. Consequently, the quality of a decision can be viewed as a function of the characteristics of the person making the decision, the quality of the information they have at their disposal, their biases, and the environmental context in which they operate.

Practically, the theory of bounded rationality can be adopted to develop an understanding of how two individuals can arrive at different conclusions on a given problem, even when accessing the same information on which to base their decision. In doing so, it also allows for specific insight into the nature of differences between human and machine in decision-making. Perhaps more importantly, it advocates the importance of recognising that humans don't always have access to all relevant information and as a result will never be perfect decision-makers.

Much research exists highlighting the limitations of humans in various decision-making problems, particularly comparative to the performance of algorithms as adopted by machines [8]. This work has been conducted in areas as diverse as candidate selection for employment interviews [6] to investment decision making [7]. However, it is notable that differences between humans and machines can be at least partially explained due to the notion that they may fundamentally consider the same problem in different ways and as a consequence, may be measuring philosophically different concepts altogether.

\section{AN APPLIED EXAMPLE}

\subsection{Boundary \#1}

To illustrate bounded rationality in an applied sport science context, the example of evaluating the match performance of a footballer can be used. To assist with the example, we consider three boundaries. Included within Boundary \#1 are items that would typically be considered in a coach's evaluation of a player (Figure 1, left). Depending on the coach, these items are likely to be numerous and varied. Items may include the number of successful passes made, the player's positioning during set pieces or their volume of sprinting completed. Some of these items may also be contextual, such as the player's age, experience level or position played. For instance, a coach may have higher expectations of more experienced players comparative to rookies, or may be more empathetic with an athlete who makes errors when playing in an unaccustomed position. Other items may even be considered subconsciously by the coach, or may be difficult to quantify, such as the player's character or behaviour. 
Limitations exist with respect to the volume of data that can be processed, interpreted and acted upon by the coach in undertaking this evaluation comparative to that of a machine, even when the evaluation is undertaken retrospectively. George Miller [9] famously proposed the 'magical number seven' to illustrate the limitations on the number of pieces of information that a human can consider when evaluating a problem, before their cognitive limitations render the addition of any new data unusable. Since, it has been suggested that the inability to process large amounts of information without the use of external aids may be an ecological adaptation to allow humans to focus on what is important, freeing up resources for other tasks [10]. The influence of these cognitive and environment constraints is substantially reduced or removed altogether for machines (Figure 1, right). By comparing these two contrasting methods, the fundamental differences between machines and humans can be explored with respect to the volume of information utilised, the way patterns in information are uncovered and interpreted and the limitations of both (what can a human see that a machine cannot - and vice versa). Obtaining feedback on these processes also helps both the machine and the human to learn and improve subsequent performance.
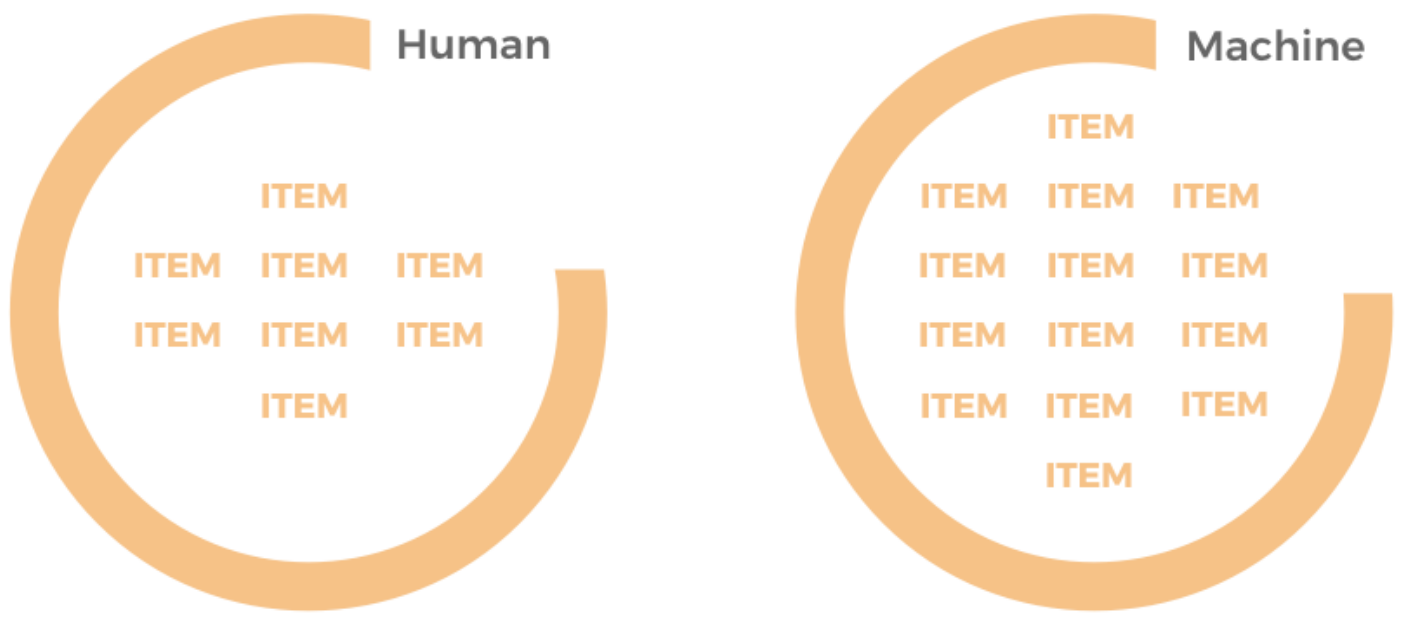

Figure 1. Differences in information utility of humans and machines when addressing a structured problem. Machines are capable of processing larger amounts of information, as well as determining dynamic, non-linear relationships between items. As the manner in which humans and machines address complex problems can differ considerably, it may not be suitable to directly compare their performance in such instances.

A pertinent example comes from Italian football, whereby player ratings made by media pundits were compared with those determined using objective models [11]. Findings revealed that the human raters utilised only a small sub-sample of 150 available data types on which to base their evaluation of a player's performance. They also revealed a particular emphasis of the humans on certain data types (for instance strikers were rated almost exclusively on the number of goals they scored). Similar results have been noted in Australian football, whereby just two indicators of ball possession (kicks and handballs) explained almost all of the variance in player match ratings assigned to midfielders by expert raters [12]. If the only information that was necessary to fully understand a problem was found within the confines of Boundary \#1, it is evident that due to the superior processing power of computers, humans are best served by seeking to automate the decision making process wherever possible. 


\subsection{Boundary \# 2}

In most of the challenging problems that humans face however, information exists that would ideally be collected and considered, but for various reasons, is not. Such items reside within the blue Boundary \#2 (Figure 2). Items may exist within this circle for a variety of reasons relating to their environmental (i.e., insufficient resources, time or interest to consider the item) or cognitive properties (i.e., non-comprehension by the user) [3]. In player evaluation, these might include items such as the leadership qualities displayed by the player during a game (not considered due to lack of reliable and valid measurement tool) or certain metrics derived from a player's tracking data (not considered due to the time taken to analyse the data and lack of interpretability by key stakeholders).

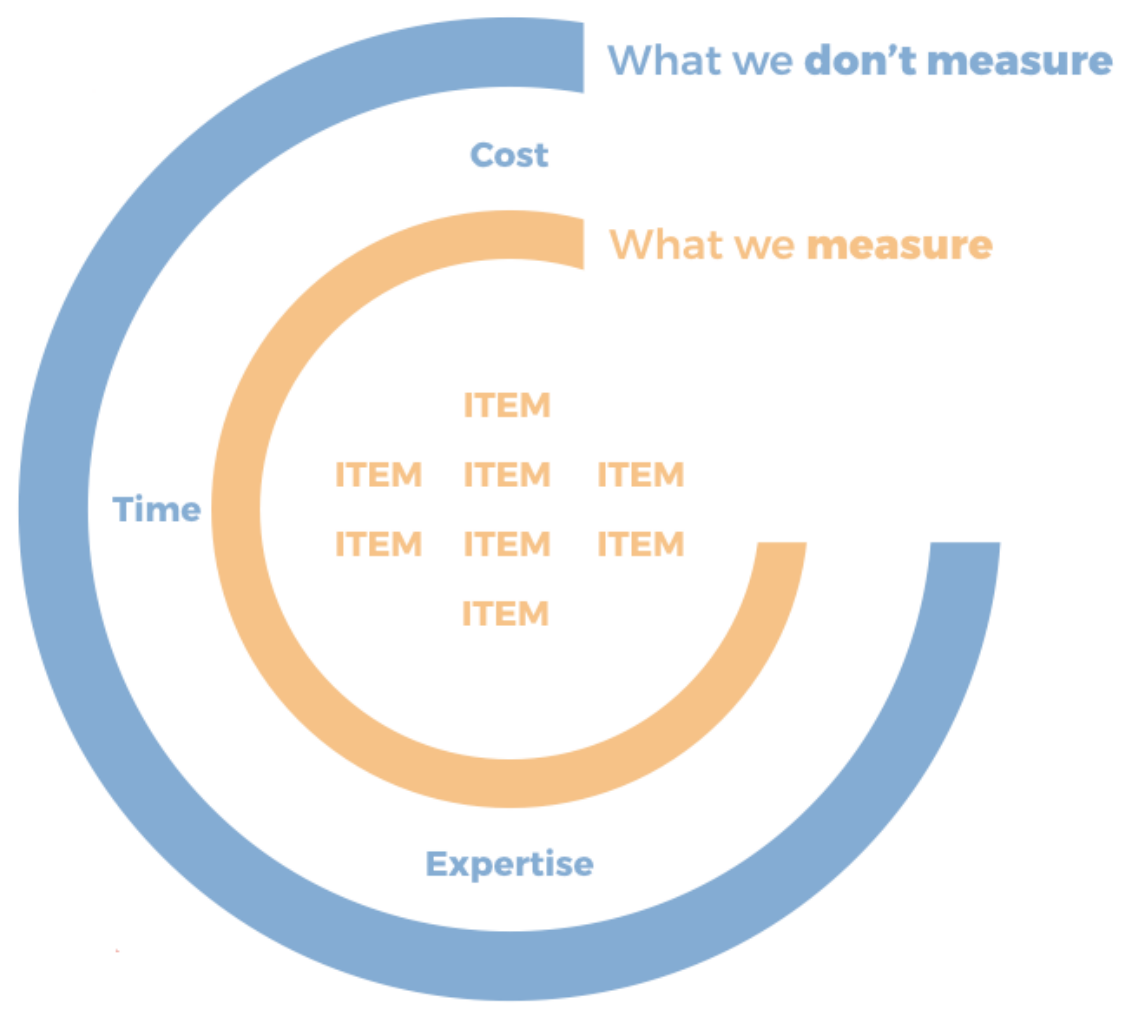

Figure 2. Environmental and cognitive constraints acting on the decision-making processes of the performance analyst. Certain items, whilst important, may not be considered due to a lack of time, insufficient resources or lack of expertise on behalf of the individual.

Thus, Boundary \#2 also provides an illustration of how humans typically settle on solutions that they find satisficing. Satisficing describes the tendency of humans to "decide on and pursue a course of action that will satisfy the minimum requirements necessary to achieve a particular goal" [13]. Thus, satisficing is related to expectation; it sees humans cease searching for alternatives to solutions once their a priori aspiration is fulfilled. A number of other concepts specific to the football evaluation example also relate to Boundary \#2. These are discussed below: 


\subsubsection{Diminishing returns}

Each additional item added to Boundary \#1 \& 2 are unlikely to provide equal improvements to improve the evaluation. The extent to which an item adds to or improves an evaluation or understanding is often referred to as information gain. The concept of diminishing returns advocates that the emphasis should be on those items contributing most strongly to the evaluation. For instance, whereby an entire team of players require evaluation on their performance immediately post-match, the coach may choose to focus on only the most important few variables on which to make the assessment.

\subsubsection{Parsimony}

Relating to diminishing returns, parsimony refers to finding an appropriate balance between collecting enough information to sufficiently inform the evaluation, contrasted against collecting many pieces of information that provide only incremental improvement to the assessment. For instance, how much more accurate or useful is the evaluation of a player's match performance following the additional consideration of their positional tracking data?

\subsubsection{Overfitting}

Relating to parsimony, limiting the pieces of information considered when addressing a question to ensure that any eventual solution remains generalisable to future similar scenarios helps to avoid overfitting. For example, consideration of an excessively high number of items in the player evaluation may mean that direct comparison with many other players competing elsewhere in other clubs or leagues may not be possible, due to a lack of widespread use of such items.

\subsubsection{Bias}

A multitude of human cognitive biases exist [see 14]. In availability bias for example, the coach may overemphasise the importance of information that they currently collect, thus inadvertently disregarding potentially important information that may be collected elsewhere. In recency bias, more recent events may be disproportionately emphasised by the coach, to the expense of a more valid long-term trend.

It is reasonable to expect that with increasing resources, technology and resulting knowledge, items that currently find themselves in Boundary \#2, will at some point in the future shift to within the borders of Boundary \#1.

\subsection{Boundary \# 3}

Boundary \#3 (Figure 3) relates to information that not only has not been considered, but also those items about which the user has no awareness. This ignorance can apply to both the human and the machine. In the abovementioned player evaluation example, this is often a problem when the coach 'doesn't know what good looks like'. Uptake of new technologies as well as the search for competitive advantage will drive the expansion of this boundary by reducing environment and cognitive constraints, creating new insights and variables worthy of consideration. 
In summary, Boundary \# 3 helps us to understand that to many of the problems faced by the sport science, an optimal solution often does not and may never exist $[3,15]$. In many problems, the limits of Boundary \#3 are unknown and potentially infinite, thus when adding new items for consideration the emphasis should be on solution improvement, rather than perfection. Since every piece of relevant information will never be considered, even the best solutions to problems will retain some error or bias because of these omissions. Hence, the process of obtaining an improved solution to a specific problem should be viewed as inductive [16].

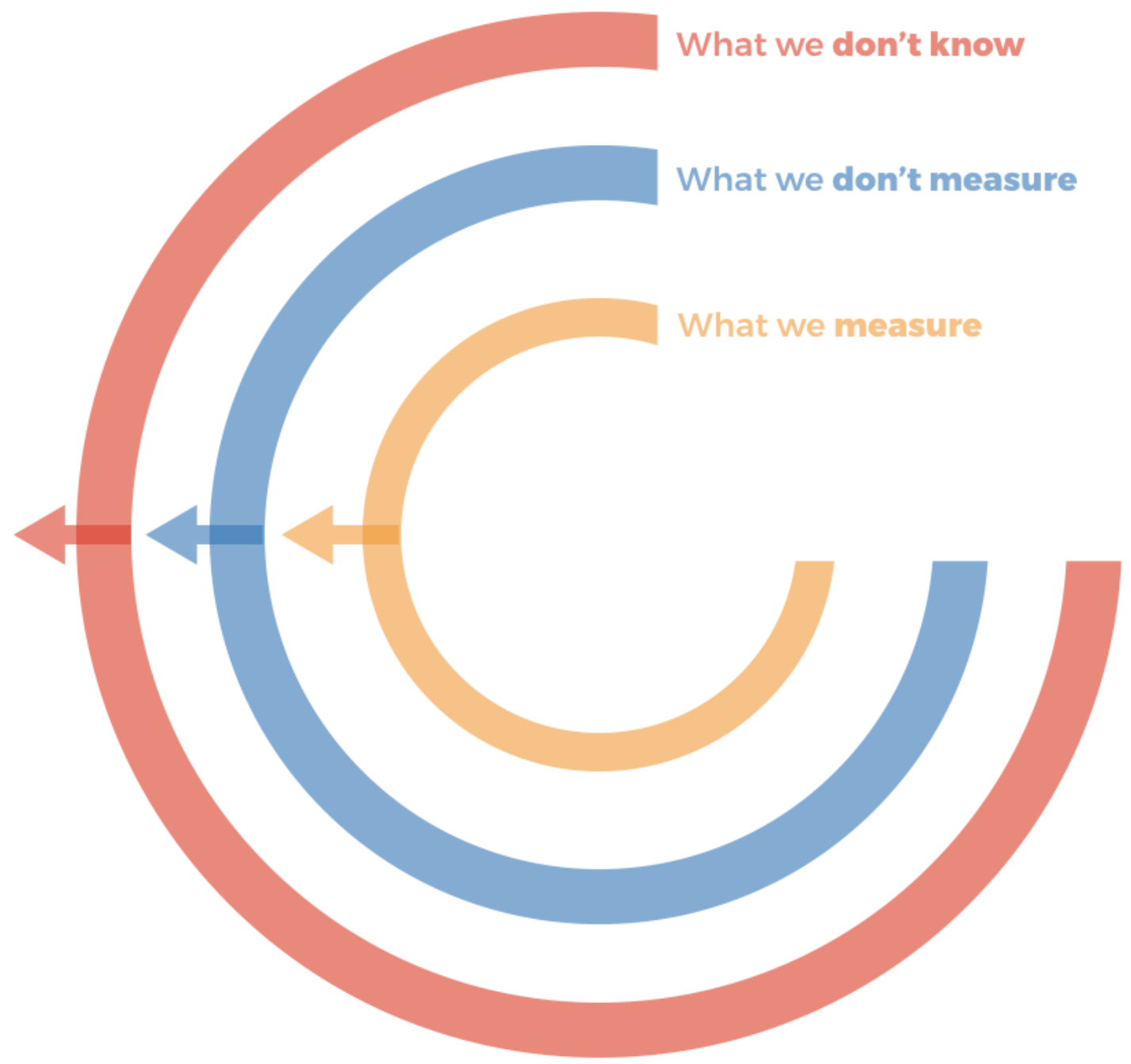

Figure 3. Visual representation of bounded rationality. Relaxation of environment and cognitive constraints increase the circumference of each circle. Uptake of new technologies as well as the search for competitive advantage will drive these expansions.

So, what learnings should the sport scientist take from bounded rationality on matters of judgement and decision-making? First, 'know what you don't know'. In the event that a differing view is presented on a certain problem, the other individual may well be in the wrong - however, they may also just be considering the same problem in a different way. The benefit of the application of machines to such problems, is that in the event that useful and accurate solutions are created, the exact mechanisms behind the success can be 
Bounded rationality revisited: Making sense of complexity in applied sport science spaces

queried, systematically evaluated and learnt from. This is much more difficult to determine in humans. Second, develop an awareness that what is an appropriate solution to a certain problem today, may no longer be sufficient in future. Third, when faced with complex problems containing relatively large number of constraints, consider offloading these to the better-suited capabilities of machines.

\section{CONCLUSION}

In the pursuit of evidence upon which to base sports science decision-making, new technologies have enabled the collection of ever-increasing volumes of data. This has the potential to deepen insights into the complexities of sport science problems. However, this may create a tendency to believe that optimal solutions to many of these problems lies in the data that is currently collected. A bounded rationality approach suggests that for many of these problems, optimal is unattainable. Decisions made assuming a mono-causality relationship between a single variable and an outcome ignores the complex interactions between variables. This simplistic manner of decision-making is often due to human limitations and may be subsumed by the transfer of these tasks from humans to machines. This article proposes bounded rationality as a theoretical framework to understand processes of decision-making in applied sport science. The nature of these decisions will continue to change as data quality and volume increases. It is proposed that by acknowledging the limitations of human information processing capacities and the lenses through which information is filtered, a humility can be engendered that can turn more of these decisions into better ones.

\section{ACKNOWLEDGEMENTS}

Neither author have any funding sources or conflicts of interest to declare.

\section{REFERENCES}

1. Simon HA. Models of man. New York: John Wiley; 1957

2. Kahneman D. Maps of bounded rationality: psychology for behavioral economics. Am Econ Rev, 2003;93:1449-75.

3. Gigerenzer G, Selten R. Bounded rationality: the adaptive toolbox. Boston: MIT press; 2002

4. Gama J. Data stream mining: the bounded rationality. Informatica. 2013;37:21-5.

5. Simon HA. Rational choice and the structure of the environment. Psychol Rev.1956;63:129.

6. Kang SK, DeCelles KA, Tilcsik A, Jun S. Whitened resumes: race and self-presentation in the labor market. Adm Sci Q. 2016;61,469-502.

7. Nofsinger JR, Patterson FM, Shank CA. Decision-making, financial risk aversion, and behavioral biases: the role of testosterone and stress. Econ Hum Biol. 2018;29,1-16.

8. Grove WM, Zald DH, Lebow BS, Snitz BE, Nelson C. Clinical versus mechanical prediction: a metaanalysis. Psychol Assess. 2000;12:19-30

9. Miller GA. The magical number seven, plus or minus two: some limits on our capacity for processing information. Psychol Rev. 1956;63:81-97.

10. Gigerenzer G. Gut feelings: The intelligence of the unconscious. New York: Penguin; 2007

11. Pappalardo L, Cintia P, Pedreschi D, Giannotti F, Barabasi AL. Human perception of performance. arXiv preprint arXiv:1712.02224:2017 
Bounded rationality revisited: Making sense of complexity in applied sport science spaces

12. McIntosh S, Kovalchik S, Robertson S. Comparing subjective and objective evaluations of player performance in Australian Rules football. PloS One. 2019; 14(8):e0220901

13. Gigerenzer G, Goldstein DG. Reasoning the fast and frugal way: models of bounded rationality. Psychol Rev. 1996;103:650-69.

14. Baron J. Thinking and deciding. 4th ed. New York: Cambridge University Press; 2007

15. Davids K, Araújo D. The concept of 'Organismic Asymmetry' in sport science. J Sci Med Sport. 2010;13:633-40.

16. Arthur WB. Inductive reasoning and bounded rationality. Am Econ Rev. 1994;84:406-11. 